\title{
Junge Geflüchtete gesellschaftlich integrieren
}

\author{
Konzeptionelle Anforderungen an Unterstützungsmaßnahmen
}

Junge männliche Geflüchtete sind in den Medien und der politischen Diskussion wiederkehrend als eine problematische Gruppe dargestellt worden, insbesondere im Hinblick auf Gewalt- und Sexualdelikte sowie Drogenhandel und Drogenkonsum. In der sozialwissenschaftlichen Diskussion wurde diesbezüglich gezeigt, dass es sich hierbei um eine vorurteilsgeleitete Dramatisierung handelt, die das Ausmaß des Problems überzeichnet und an kulturrassistische Stereotype appelliert.

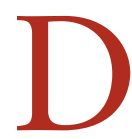
emgegenüber haben Analysen der verfügbaren Daten gezeigt, dass bei den polizeilich erfassten Tatverdächtigen nur bestimmte Teilgruppen junger Geflüchteter ${ }^{1}$ und diese nur in geringem Maße im Vergleich zu altersgleichen Einheimischen auffällig sind (Feltes et al. 2017; Hörnle 2018; Pfeiffer et al. 2018). Aus der notwendigen Kritik pauschaler Darstellungen junger männlicher Geflüchteter als Problemgruppe (Gruhlich 2019; Schartau et al. 2018) kann aber nicht die Konsequenz gezogen werden, auf eine Auseinandersetzung mit den Ursachen problematischer

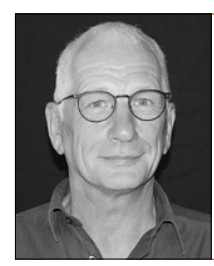

\section{Albert Scherr}

Pädagogische Hochschule Freiburg, Freiburg, Deutschland *1958; Prof. Dr. habil., Direktor des Instituts für Soziologie der Pädagogischen Hochschule Freiburg.

scherr@ph-freiburg.de

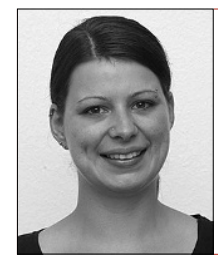

\section{Helen Breit}

Pädagogische Hochschule Freiburg, Freiburg, Deutschland *1987; Soziale Arbeit (BA) und Erziehungswissenschaft (MA); wissenschaftliche Mitarbeiterin an der PH Freiburg/Institut für Soziologie.

helen.breit@ph-freiburg.de

Zusammenfassung Grundlage des Beitrags ist ein qualitatives Forschungsprojekt zu krisenhaften Entwicklungsverläufen und problematischen Verhaltensweisen bei jungen Geflüchteten. Ausgehend von einer knapp gefassten Kritik politischer und medialer Dramatisierungen, die junge männliche Geflüchtete als bedrohliche Problemgruppe darstellen, werden Überlegungen zu Anforderungen an eine Soziale Arbeit dargestellt, die darauf ausgerichtet ist, eine dem Bedarf junger Geflüchteter angemessene Unterstützung zu ermöglichen.

Schlüsselwörter Junge Geflüchtete, Flüchtlingssozialarbeit, Integration, Unterstützungsstrukturen, Professionalität
Entwicklungsverläufe und Verhaltensweisen von Geflüchteten zu verzichten und eine Diskussion darüber zu führen, was Möglichkeiten und Erfordernisse der Verbesserung von Maßnahmen sind, die das Risiko von Delikten und selbstschädigenden Praktiken verringern. In diesem Beitrag geht es dabei zentral um Gestaltungserfordernisse und -möglichkeiten der Jugendhilfe und der Flüchtlingssozialarbeit innerhalb der gegebenen politischen und rechtlichen Rahmenbedingungen.

\section{Warum einige Geflüchtete scheitern}

Mit dieser Zielsetzung haben wir im Forschungsprojekt „Gescheiterte Flüchtlinge. Problemlagen und Unterstützungsmöglichkeiten "2 Befunde der internationalen Forschung (s. u. a. ICMPD 2019; ISMU 2019; Kury et al. 2018; Lechner und Huber 2017) aufgearbeitet sowie qualitative Interviews mit Fachkräften (insbesondere der Flüchtlingssozialarbeit, der Jugendarbeit und Jugendhilfe) sowie mit jungen Geflüchteten geführt, bei denen problematische Entwicklungen vorlagen. Fasst man das Ergebnis unserer Analyse grob zusammen, dann lautet der zentrale Befund, dass problematische Entwicklungen nur dann angemessen verstanden werden können, wenn das Zusammenwirken von biografischen Erfahrungen und Belastungen von Geflüchteten, Integrationsanstrengungen von Geflüchteten und Integrationsanforderungen der Aufnahmegesellschaft, rechtlichen Einschränkungen ihrer Teilhabemöglichkeiten sowie Umfang und Qualität von Unterstützungsmaßnahmen in den Blick genommen wird. Junge Geflüchtete wollen sich gesellschaftlich integrieren. Sie müssen dazu aber erhebliche Anforderungen bewältigen, nicht zuletzt in den Bereichen Spracherwerb sowie der schulischen und beruflichen Qualifizierung. Und dies zum Teil unter ungünstigen Voraussetzungen wie einer geringen oder fehlenden schulischen Vorbildung und erheblicher 
psychischer Belastungen. Sie sind dann auf eine umfassende Unterstützung ihrer Integrationsanstrengungen angewiesen, die in vielen Fällen nicht zuletzt deshalb positiv verlaufen, weil Geflüchtete dafür erhebliche Eigenleistungen erbringen und bei Schwierigkeiten angemessene Unterstützung durch Sozialarbeiter_innen oder andere Bezugspersonen finden (Scherr und Breit 2020). Wenn Integrationsanstrengungen jedoch durch einen unsicheren Aufenthaltsstatus und eine fehlende Bleibeperspektive erschwert und ggf. entmutigt werden, schulische und berufliche Anforderungen aufgrund eines zu hohen Zeit- und Erfolgsdrucks als überfordernd erlebt werden sowie erforderliche sozialarbeiterische, pädagogische oder therapeutische Unterstützung rechtlich verstellt oder faktisch nicht zugänglich ist, kann dies zu krisenhaften Verläufen führen, die die Wahrscheinlichkeit von Alkohol- und Drogenkonsum und strafrechtlichen Delikten erhöhen.

Um die damit nur sehr grob skizzierten Zusammenhänge genauer in den Blick zu nehmen, ist eine komplexe Analyse erforderlich, die eine Betrachtung der fallspezifischen biografischen Verläufe vor, während und nach der Flucht mit einer differenzierten Untersuchung der rechtlichen und institutionellen Bedingungen nach der Einreise sowie der im jeweiligen lokalen Kontext gegebenen Struktur von Qualifizierungs- und Unterstützungsmaßnahmen verbindet. Unsere Ergebnisse können hier nicht umfassend dargestellt werden. Im Folgenden beschränken wir uns darauf aufzuzeigen, welche zentralen Gesichtspunkte wir aus unserer Forschung ableiten können, die aus sozialwissenschaftlicher Sicht als leitende Prinzipien bei einer konzeptionellen Weiterentwicklung von Unterstützungsmaßnahmen Berücksichtigung finden sollten.

\section{Kontinuität und Kohärenz}

Flüchtlingsbiografien sind, insbesondere bei unbegleiteten Minderjährigen ${ }^{3}$ und jungen Erwachsenen, vielfach durch Erfahrungen mit Gewalt und Misshandlung vor und während der Flucht, lang andauernde Unsicherheit bzgl. der Zukunftsperspektive, Beziehungsabbrüche sowie fehlende oder nur medial aufrechterhaltene familiale Beziehungen gekennzeichnet. Es ist keineswegs außergewöhnlich, dass in Interviews davon berichtet wird, dass Eltern oder Verwandte im Herkunftsland ermordet wurden, Freunde und Reisegefährten die Flucht nicht überlebt haben oder die Interviewten während der Flucht selbst inhaftiert und misshandelt wurden. Daraus resultieren psychische Verletzungen, die zu einem starken Bedürfnis nach emotionalen Bindungen führen, es aber zugleich erschweren können, anderen Personen zu vertrauen. Deshalb sind Sozialarbeiter_ innen für Geflüchtete potenziell wichtige Bezugspersonen, von denen nicht nur praktische Unterstützung erwartet wird, sondern zu denen Nähe-Verhältnisse gesucht werden. Deshalb kommt dem Aufbau vertrauensgestützter professioneller Beziehungen sowie der Kontinuität und Kohärenz von Unterstützungsangeboten bei jungen Geflüchteten eine besonders hohe Bedeutung zu. Erforderlich sind Formen der Unterstützung durch Fachkräfte, zu denen eine vertrauensgestützte Beziehung aufgebaut werden kann, was ersichtlich Zeit benötigt, und die als langfristig angelegte Begleitung von Entwicklungsprozessen angelegt sind. D. h.: Anzustreben ist eine umfassende und kontinuierliche Unterstützung, die darauf angelegt ist, bei Bedarf immer wieder erneut zu versuchen, Auswege aus Krisen aufzuzeigen und Entwicklungschancen zu eröffnen sowie zur Stärkung des psychischen Wohlbefindens, der Entwicklung und Aufrechterhaltung eines positiven Selbstbildes beizutragen sowie Gefühle der Sicherheit, Geborgenheit und der Stabilität zu ermöglichen. Die Bedeutung von stabilen personellen Beziehungen resultiert auch daraus, dass Geflüchteten die Logik ausdifferenzierter institutioneller Zuständigkeiten aus ihren Herkunftsländern ggf. nicht bekannt ist und sie deshalb Schwierigkeiten haben, nachzuvollziehen, weshalb sie sich an andere Personen wenden sollen als diejenigen, die sie als hilfsbereite Bezugspersonen bereits kennengelernt haben.

Damit kommt Erstkontakten, z. B. in stationären Einrichtungen der Jugendhilfe oder Erstaufnahmeeinrichtungen, eine Schlüsselrolle für den weiteren Prozessverlauf zu. Sie repräsentieren für Geflüchtete zudem auch zentral, mit welchen Formen der professionellen Unterstützung sie in der Aufnahmegesellschaft rechnen können. Verweisungen an Fachdienste und Beziehungsabbrüche, die sich aus wechselnden institutionellen Zuständigkeiten ergeben, können jedoch nicht vermieden werden. Es bedarf deshalb eines Bewusstseins der Fachkräfte dafür, dass diese Wechsel einen immer wieder erneuten Aufbau der Vertrauensbasis erfordern, die für sozialarbeiterische Arbeitsbündnisse unverzichtbar ist. Deshalb sollten Übergänge zwischen Institutionen und Prozesse der Verweisung an Fachdienste möglichst durch Bezugspersonen begleitet werden, zu denen eine vertrauensgestützte Beziehung entstanden ist. Eine bloße Weitergabe von Informationen über Institutionen und Zuständigkeiten kann dagegen dazu führen, dass Geflüchtete nicht in der Lage sind, aktiv Unterstützung bei ihnen bislang unbekannten Personen einzufordern und dann zudem ggf. unter den Verdacht gestellt werden, eine erwartbare Mitwirkung im Hilfeprozess ohne Not zu verweigern. 


\section{Geflüchtete sind auch Jugendliche}

Auch die unzureichende Berücksichtigung der Bedarfe von jungen Geflüchteten kann das Risiko von krisenhaften Entwicklungs- und Integrationsverläufe erhöhen. Denn sie können nicht auf ihre Flüchtlingseigenschaft reduziert werden, sondern sind auch Jugendliche und junge Erwachsenen, die lebensphasentypische Entwicklungskrisen bewältigen müssen. Hinzu kommen ggf. zudem erhebliche psychische Belastungen sowie eine Situation der existenziellen Unsicherheit während des Asylverfahrens. Im Fall von UMA kommt die Notwendigkeit hinzu, sich mit einer Situation auseinanderzusetzen, die durch Trennung von der Herkunftsfamilie bei vielfach zugleich fortbestehenden emotionalen Bindungen und ggf. finanzielle Verpflichtungen gekennzeichnet ist.

Nach der Ankunft in Deutschland benötigen junge Geflüchtete deshalb einen Kontext, in dem sie sich sicher fühlen können und in denen ihnen die Zeit zugestanden wird, die sie benötigen, um sich emotional zu stabilisieren und auf das Leben in der Aufnahmegesellschaft einzustellen. Deshalb ist eine Perspektive, die auf die möglichst schnelle schulische und berufliche Integration fokussiert ist, unzureichend. Diese wird der Sozialen Arbeit zwar durch rechtliche Vorgaben nahelegt, die Versuche veranlassen, durch schulische und berufliche Integrationsleistungen zur Ermöglichung einer aufenthaltsrechtlichen Bleibeperspektive beizutragen, wenn geringe Aussichten auf eine flüchtlingsrechtliche Anerkennung bestehen. In unserer Forschung und in internationalen Studien hat sich jedoch gezeigt, dass dies zu einer Vernachlässigung von Erfordernissen der Persönlichkeitsentwicklung und damit zu einer Situation führen kann, die als Überforderung erlebt wird und ggf. den kontraproduktiven Effekt von Schul- und Ausbildungsabbrüchen hat.

Aufgrund vielfach schwieriger biografischer Voraussetzungen (psychische Belastungen, keine oder geringe schulische Bildung), der Notwendigkeiten des Spracherwerbs, der Qualifizierung und sozialen Verortung in der Aufnahmegesellschaft sowie der Bewältigung asyl- und aufenthaltsrechtlicher Verfahren kann von jungen Geflüchteten zudem kein krisenfreier biografischer Verlauf und kein geradliniger und schneller Integrationsprozess erwartet werden. Vielmehr ist auch bei ihnen von jugendtypischen Formen des abweichenden Verhaltens, biografischen Krisen und Phasen der Re-Orientierung auszugehen sowie davon, dass sie als neu Zugewanderte erhebliche Herausforderungen bei der Entwicklung einer tragfähigen Lebensperspektive bewältigen müssen. Sozialarbeiterische Unterstützungsangebote sind deshalb erstens darauf verwiesen, eine Perspektive auf junge Geflüchtete einzunehmen, die auf eine umfassende
Unterstützung bei der Bewältigung von Entwicklungsund Integrationsbedarfen ausgerichtet ist. Um Risiken des Scheiterns zu verringern, ist es zweitens erforderlich, jungen Geflüchteten ausreichende Zeit für ein Ankommen und eine Orientierung in der Aufnahmegesellschaft sowie die Bewältigung von Entwicklungsaufgaben und Integrationsanforderungen zuzugestehen. Diesbezüglich können Fachkräfte der Jugendhilfe und Flüchtlingsarbeit zwar die aufenthalts- und flüchtlingsrechtlichen Rahmenbedingungen nicht ignorieren. Gleichwohl können sie versuchen, in den von ihnen verantworteten Bereichen keinen überfordernden Zeit- und Erfolgsdruck zu erzeugen sowie andere Institutionen, Schulen, Betriebe und die Öffentlichkeit für die vielfältigen Anforderungen zu sensibilisieren, mit denen junge Geflüchtete konfrontiert sind. Zudem können die mit jungen Geflüchteten befassten Institutionen der Sozialen Arbeit fachpolitisch für eine solche Ausgestaltung der gesetzlichen und institutionellen Bedingungen eintreten, die darauf ausgerichtet ist, eine Überforderung durch Zeit- und Qualifizierungsdruck, insbesondere bei jungen Geflüchteten mit ungünstigen Bildungsvoraussetzungen und mit erheblichen psychosozialen Belastungen, zu vermeiden.

\section{Keine Normalbiografien}

Die Leistungen der Kinder- und Jugendhilfe sind auch bei jungen Geflüchteten an die Altersgrenzen gebunden, mit denen Kinder und Jugendliche von Erwachsenen unterschieden werden. Diesen Festlegungen liegen Annahmen über Entwicklungsverläufe und damit verknüpfte Erwartungen im Hinblick auf die altersgemäße Entwicklung von Kompetenzen und Bewältigung von Entwicklungsaufgaben zugrunde, deren Grundlage deutsche oder europäische Normalbiografien sind. Im Fall von jungen Geflüchteten sind diese Annahmen jedoch aus einer Reihe von Gründen problematisch: Sie waren zum Teil bereits vor der Flucht sowie als unbegleitete Minderjährige während der Flucht darauf verwiesen, in einem Ausmaß selbstständig und eigenverantwortlich zu leben, das hierzulande nur Erwachsenen zugetraut wird. Zugleich aber war ihnen vielfach die Möglichkeit der sozialen, emotionalen und kognitiven Entwicklung in Familien und in Schulen verstellt, die hierzulande gewöhnlich als Voraussetzungen der Persönlichkeitsentwicklung gelten. Insofern ist bei jungen Geflüchteten gerade nicht von einer nach deutschen Maßstäben altersgemäßen Entwicklung auszugehen, sondern davon, dass sie in manchen Hinsichten älter, in anderen aber zugleich jünger sind als dies gewöhnlich bei altersgleichen und seit ihrer Kindheit in Deutschland aufgewachsenen jungen Menschen der Fall ist. 
Ansprüche auf Leistungen sollten deshalb nicht am biologischen Alter, sondern an den je individuellen Bedarfslagen orientiert sein. Dies betrifft insbesondere die Gewährleistungen von Hilfen zur Erziehung (\$27ff. SGB VIII) nach der Vollendung der Volljährigkeit bzw. des 21. Lebensjahres, da diese das einzige Unterstützungsangebot mit einer umfassenden Zuständigkeit sind und zugleich eine intensive Begleitung junger Geflüchteter ermöglichen, die in dieser Weise in Maßnahmen der Flüchtlingssozialarbeit mit Erwachsenen nicht gegeben ist. Der in der Fachdiskussion konsensuelle Befund, dass problematische Entwicklungsverläufe bei jungen unbegleiteten Geflüchteten insbesondere nach dem Ausscheiden aus den Hilfen zur Erziehung virulent werden, verweist deshalb darauf, dass jungen Geflüchteten ggf. eine Verselbstständigung zu einem Zeitpunkt zugemutet wird, zu dem sie zu einer eigenverantwortlichen Lebensführung unter den Bedingungen der Aufnahmegesellschaft noch nicht zureichend befähigt sind. ${ }^{4}$ Folglich ist in der Kinder- und Jugendhilfe sowie der Migrationssozialarbeit eine Diskussion dazu erforderlich, ob und ggf. wie Leistungen der Hilfen zur Erziehung bei jungen Geflüchteten über das 21. Lebensjahr hinaus erbracht, oder durch eine Ausweitung integrationspolitischer Maßnahmen (etwa auf Grundlage des $\$ 45$ Aufenthaltsgesetz) ersetzt werden können. Dies ist auch im Hinblick auf solche junge Geflüchtete bedeutsam, die als junge Erwachsene nach Deutschland einreisen und in der Folge nicht zu Adressat_innen der stationären Jugendhilfe werden.

Bei einem Teil der jungen Geflüchteten werden Hilfen zur Erziehung zudem bereits mit dem Erreichen der Volljährigkeit beendet, weil Jugendämter zu der Einschätzung gelangen, dass die für eine Verlängerung bis zum 21. Lebensjahr erforderlichen Mitwirkungspflichten nicht erfüllt werden. Damit fallen aber gerade auch solche junge Geflüchtete aus den Hilfen zur Erziehung, deren Entwicklung in besonderer Weise problematisch verläuft. Deshalb sollte eine Beendigung von Hilfen zur Erziehung aufgrund einer Nichterfüllung der sog. Mitwirkungspflichten im Fall von jungen Geflüchteten nur erfolgen, wenn nach einer sehr umfassenden und sorgfältigen Analyse der fallspezifischen Problematik die Einschätzung begründet werden kann, dass keinerlei Aussicht darauf besteht, mittelfristig eine tragfähige Grundlage für eine sozialarbeiterische Arbeitsbeziehung herzustellen. Diesbezüglich sehen wir einen Bedarf an einer Erweiterung des bestehenden Angebotsspektrum sowie der Entwicklung flexibler Hilfekonzepte, die dazu beitragen, Abbrüche in den Hilfen zur Erziehung zu vermeiden und als niederschwellige Hilfen konzipiert sind.

\section{Hilfen für junge Erwachsene}

Ein innovativer Ansatz, um im Bedarfsfall ein faktisches Herausfallen ehemaliger unbegleiteter Minderjähriger aus Unterstützungsmaßnahmen der Jugendhilfe zu vermeiden, wurde in unserer Forschung in Projekten sichtbar, die sich vor allem an solche volljährig Gewordene richten, die nicht länger in der stationären Jugendhilfe verbleiben können und zugleich von Unterstützungsangeboten mit einer Komm-Struktur nicht erreicht werden. Um die erforderliche Unterstützung weiter gewährleisten zu können, wurden in einem Fall in einer Gemeinschaftsunterkunft eigene Wohngruppen für junge Geflüchtete eingerichtet. In einem anderen Fall waren Bestrebungen zu verzeichnen, jungen Geflüchteten Möglichkeiten sozialpädagogisch begleiteter Wohnformen ( $\$ 13$ Absatz 3 SGB VIII) anzubieten und entsprechende Angebote zu etablieren, die auf einen angespannten Wohnungsmarkt, Abbrüche von Hilfen zur Erziehung sowie angestrebte berufliche Qualifizierung in Verbindung mit einem Bedarf an sozialarbeiterischer Begleitung reagieren.

Ein anderer Ansatz zur Gewährleistung einer angemessenen Unterstützung auch für junge Erwachsene zeigte sich in einem Modellprojekt, das aufsuchende Jugendsozialarbeit in einer Gemeinschaftsunterkunft realisierte. Ziel war es, ältere Jugendliche und junge Erwachsene durch aufsuchende Arbeit in der Gemeinschaftsunterkunft zu erreichen und dann in Regelangebote der Jugendarbeit und Jugendsozialarbeit sowie an Fachstellen weiterzuvermitteln. Eine Fachkraft beschreibt auf Grundlage ihrer Erfahrungen in diesem Projekt den aufsuchenden Ansatz in Gemeinschaftsunterkünften als geeignet, um Kontakt mit der Zielgruppe herzustellen und Beziehungen aufzubauen. In einem anderen Kontext wurde als Reaktion auf eine fehlende Nachbetreuung ehemaliger unbegleiteter Minderjähriger, deren Hilfebeendigungen z. T. auf ungeplante Abbrüche zurückzuführen ist, ebenfalls ein Angebot der aufsuchenden Jugendsozialarbeit für junge Geflüchtete etabliert. Auch hier berichtet die Fachkraft davon, dass durch den Ansatz der aufsuchenden Arbeit auch diejenigen jungen Geflüchteten erreicht werden können, die von anderen Angeboten weniger bis gar nicht erreicht werden. Ziele des Angebots sind der Aufbau einer tragfähigen Arbeitsbeziehung, die Sichtbarmachung von professionellen Ansprechpersonen und eine lebensweltliche Unterstützung, die sich an den von den Adressat_innen artikulierten Bedürfnissen orientiert.

Weiter zeigt sich in unserer Analyse, dass aufsuchende und niedrigschwellige Unterstützungsangebote für diejenigen jungen Geflüchteten entwickelt und ausgebaut werden müssen, die nicht in der Lage bzw. nicht dazu 
motiviert sind, die Komm-Strukturen von Beratungsangeboten und Fachdiensten in Anspruch zu nehmen. In unserer Forschung wurde diesbezüglich deutlich, dass ein Teil der jungen Geflüchteten, die von anderen Unterstützungsangeboten nicht mehr erreicht werden, zu Adressat_innen der aufsuchenden und mobilen Jugendarbeit werden. Die fachlichen Grundsätze der mobilen Jugendarbeit stellen unseres Erachtens eine geeignete Grundlage auch für die Arbeit mit jungen Geflüchteten dar, weshalb eine Anerkennung der Ausweitung ihres Arbeitsauftrags auf die Zielgruppe „Junge Geflüchtete" geboten ist, wie sie in der Fachdiskussion auch ausdrücklich eingefordert wurde (LAG 2019).

\section{Öffnung von Regelangboten}

Angebote der Jugendarbeit stehen Geflüchteten zwar prinzipiell ebenso offen wie in Deutschland aufgewachsenen jungen Menschen. In den von uns geführten Interviews mit Fachkräften aus den Bereichen der Regelangebote zeigt sich jedoch, dass die Zugänglichkeit faktisch nicht problemlos und selbstverständlich gewährleistet ist (vgl. Deinet und Scholten 2019; Scherr/Sachs 2020). Vielmehr sind gezielte Maßnahmen erforderlich, um junge Geflüchtete als Zielgruppe anzusprechen und $\mathrm{Zu}$ gangshürden abzubauen.

Die Fachkräfte, denen eine tatsächliche Öffnung gelungen ist, weisen diesbezüglich insbesondere auf die Notwendigkeit hin, jungen Geflüchteten die Arbeitsweise und die Zielsetzungen der Jugendarbeit verständlich zu machen, da diesen Formen der offenen, aufsuchenden und mobilen Jugendarbeit aus ihren Herkunftsländern vielfach nicht bekannt sind. Zudem sind für die Öffnung konzeptionelle Klärungsprozesse sowie eine Qualifizierung zu rechtlichen Fragen bei den Fachkräften ebenso erforderlich wie ggf. der Abbau von Abwehrhaltungen und Vorurteilen bei denjenigen, welche die Angebote bereits nutzen.

Unsere Forschungsergebnisse zeigen zudem, dass der Zugang junger Geflüchteter zu den Regelangeboten der Kinder- und Jugendarbeit lokal sehr unterschiedlich gut gelingt, nicht zuletzt in Abhängigkeit davon, wie gut die Kooperationen zwischen den Trägern der Jugendarbeit, Jugendsozialarbeit, stationärer Jugendhilfe, Trägern der öffentlichen Jugendhilfe und der Flüchtlingssozialarbeit ausgeprägt sind. Maßnahmen, die darauf zielten, diesen Prozess der Öffnung durch Projektförderung und Fortbildung zu unterstützen, wurden von den Fachkräften, die diese in Anspruch genommen haben, als hilfreich beschrieben.

Die Erfahrungen der Fachkräfte weisen auch darauf hin, dass gängige institutionelle Abgrenzungen und Spezialisierungen für die Arbeit mit jungen Geflüchteten überprüft werden müssen. So wurde für die offene Jugendarbeit akzentuiert, dass der Bedarf an Unterstützung bei der Arbeits-, Wohnungs-, Schulplatz- und Ausbildungssuche hier nicht ignoriert bzw. an zuständige Fachdienste delegiert werden kann. Für die offene, aber auch für die mobile Jugendarbeit hat es sich als hilfreich erwiesen, die Zusammenarbeit mit Sozialdiensten in Gemeinschaftsunterkünften, Einrichtungen der stationären Jugendhilfe, Jugendmigrationsdiensten sowie mit sprachvorbereitenden Schulklassen auf- oder auszubauen.

\section{Sensibilität für Sprachbarrieren}

Soziale Arbeit vollzieht sich zentral als sprachliche Kommunikation. Begrenzte Kenntnisse der deutschen Sprache bei jungen Geflüchteten sind folglich eine erhebliche Beeinträchtigung der Möglichkeiten Sozialer Arbeit, Probleme und Bedarfe ihrer Adressat_innen zu verstehen und sich mit diesen darüber auseinanderzusetzen. In den geführten Interviews wurde jedoch eine eher geringe Sensibilität der Fachkräfte für diese Problematik und die Tendenz zu einem pragmatischen Umgang damit deutlich. Manche Fachkräfte bewerteten es als weitestgehend unproblematisch, dass sie teils nur rudimentär mit ihrer Klientel kommunizieren können; sie verwiesen auf die Bedeutung von Gestik und Mimik und erklärten, dass es auch möglich sei, mit technischen Hilfsmitteln (Übersetzungsprogrammen) zu kommunizieren. Andere verwiesen auf die Möglichkeit, ihre eigenen Sprachkenntnisse (häufig Französisch und/oder Englisch) einzusetzen sowie darauf, dass sie dies gegenüber dem Einsatz von Dolmetschenden präferieren. Eingeschränkt wurde dies von einigen anderen Fachkräften: zum einen bezüglich der Kommunikation über emotionale Themen, insbesondere tieferliegenden psychischen Belastungen, zum anderen in Bezug auf behördliche Angelegenheiten. Auf die Notwendigkeit einer Zusammenarbeit mit Dolmetschenden wurde insbesondere im Hinblick auf ausländerrechtlichen Fragen sowie die Übersetzung von amtlichen Bescheiden und Dokumenten der Hilfeplanung nach dem SGB VIII hingewiesen. Übersetzer_innen sind lokal jedoch in sehr unterschiedlichem Ausmaß verfügbar. ${ }^{5}$

Problematisiert wird der Einsatz von Dolmetschenden auch in Bezug auf den erhöhten Organisationsund Zeitaufwand für die Durchführung von Gesprächen. Zudem wurde auf die Schwierigkeit hingewiesen, dass die Fachkräfte keine Kontrolle darüber haben, ob das, was sie und was ihre Klient_innen kommunizieren wollen, auch tatsächlich genau übersetzt oder aber von Dolmetschenden vorinterpretiert wird sowie ob es in der genutzten Sprache auch alle wörtlichen und sinn- 
gemäßen Entsprechungen gibt. Fachkräfte sind in diesen Fällen herausgefordert, ein neues Arbeitsbündnis zu gestalten, das nicht mehr auf die klassische Konstellation Fachkraft - Klient_in begrenzt ist, sondern um Dolmetschende erweitert wird. Dies hat auch zur Folge, dass Kompetenzen und Zuständigkeiten neu verteilt und ausgehandelt werden müssen (s. auch Schweitzer 2019; Eubel 2019).

Bezüglich der Sprachfähigkeiten junger Geflüchteter wurde von Fachkräften wiederkehrend darauf hingewiesen, dass eine erfolgreiche Beendigung der Berufsausbildung häufig daran scheitere, dass die jungen Menschen ihre Ausbildung mit einem zu geringen Sprachniveau aufnahmen und aufgrund dieser fehlenden Sprachfähigkeiten den Anforderungen der Berufsschule nicht entsprechen können. Dies treffe besonders für diejenigen zu, die bei der Ankunft in Deutschland eine geringe schulische Vorbildung vorweisen konnten. Vor dem Hintergrund der skizzierten Beobachtungen sind erstens Überlegungen dazu erforderlich, wie die Unterstützung junger Geflüchteter beim Spracherwerb verbessert werden kann; zweitens besteht in der Ausund Fortbildung ein Bedarf an Maßnahmen, mit denen Sozialarbeiter_innen für die Schwierigkeiten sensibilisiert werden, die aus den Grenzen der sprachlichen Verständigungsmöglichkeiten resultieren und zu einem professionellen Umgang damit befähigt werden.

\section{Konsequenzen}

Die dargestellten konzeptionellen Anforderungen verweisen

1. auf fachliche Klärungsbedarfe in der Sozialen Arbeit,

2. auf Erfordernisse der Weiterentwicklung institutioneller Strukturen sowie

3. auf Erfordernisse einer Sozial- und Integrationspolitik, die allen jungen Geflüchteten, die mittel- und langfristig in Deutschland leben werden, Teilhabechancen eröffnet.

Diesbezüglich konnten hier nur einige generelle Gesichtspunkte aufgezeigt werden, auf die wir in unserem Forschungsbericht ausführlicher eingehen und die dort auch um weitere Aspekte, so u. a. den Bedarf an einer Verbesserung der Vernetzung und der koordinierten Fallbegleitung, ergänzt werden. ${ }^{6}$ Von einer qualitativen Verbesserung von Unterstützungsmaßnahmen ist zu erwarten, dass es jungen Geflüchteten erleichtert wird, eine tragfähige Lebensperspektive in der Aufnahmegesellschaft zu entwickeln sowie dass dadurch das Risiko problematischer Entwicklungen und Verhaltensweisen reduziert wird. Insofern handelt es sich um eine Win-
Win-Situation, wenn von einem politischen Primat des Interesses ausgegangen werden kann, junge Geflüchtete gesellschaftlich zu integrieren.

Funding. Open Access funding enabled and organized by Projekt DEAL.

Open Access. Dieser Artikel wird unter der Creative Commons Namensnennung 4.0 International Lizenz veröffentlicht, welche die Nutzung, Vervielfältigung, Bearbeitung, Verbreitung und Wiedergabe in jeglichem Medium und Format erlaubt, sofern Sie den/die ursprünglichen Autor(en) und die Quelle ordnungsgemäß nennen, einen Link zur Creative Commons Lizenz beifügen und angeben, ob Änderungen vorgenommen wurden.

Die in diesem Artikel enthaltenen Bilder und sonstiges Drittmaterial unterliegen ebenfalls der genannten Creative Commons Lizenz, sofern sich aus der Abbildungslegende nichts anderes ergibt. Sofern das betreffende Material nicht unter der genannten Creative Commons Lizenz steht und die betreffende Handlung nicht nach gesetzlichen Vorschriften erlaubt ist, ist für die oben aufgeführten Weiterverwendungen des Materials die Einwilligung des jeweiligen Rechteinhabers einzuholen.

Weitere Details zur Lizenz entnehmen Sie bitte der Lizenzinformation auf http://creativecommons.org/licenses/by/4.0/deed.de

1. Hier und im Folgenden verwenden wir den Terminus junge Geflüchtete als Sammelbezeichnung für diejenigen, die in Deutschland eine Anerkennung als Flüchtlinge anstreben sowie Kinder, Jugendliche oder junge Erwachsene sind, deren Alter im SGB VIII (\$7) der Kategorie „junge Menschen“ entspricht, für die gilt, dass sie noch nicht 27 Jahre alt sind.

2. Das Forschungsprojekt wird vom Ministerium für Soziales und Integration Baden-Württemberg unterstützt. Eine Veröffentlichung der Ergebnisse ist im ersten Halbjahr 2021 geplant.

3. Die rechtliche Terminologie des SGB VIII (\$42) verwendet die Bezeichnung unbegleitete ausländische Kinder und Jugendliche (UMA), in der Fachdiskussion der Sozialen Arbeit ist dagegen vielfach von unbegleiteten minderjährigen Flüchtlingen die Rede.

4. Im SGBV III sind verschiedene Möglichkeiten der Unterstützung junger Erwachsener gegeben, diese räumen Fachkräften z. T. aber nur einen eng gefasstem Entscheidungsspielraum ein.

5. Ggf. sind auch finanzielle Mittel unzureichend, um Dolmetschende im erforderlichen Umfang hinzuziehen.

6. Vorgeschlagen wird dort eine Anhebung der Altersgrenzen bei den Möglichkeiten zur Aufenthaltssicherung für gut Integrierte Heranwachsende (\$25a Aufenthaltsgesetz) sowie eine stärkere aufenthaltsrechtliche Anerkennung schulischer und sprachlicher Qualifizierungsanstrengungen.

\section{Literatur}

Deinet, U., \& Scholten, L. (2019). Schnelle Reaktion der Offenen Kinderund Jugendarbeit auf die neuen Zielgruppen der Kinder und Jugendlichen mit Fluchthintergrund. In U. Deinet (Hrsg.), Herausforderung angenommen. Offene Kinder- und Jugendarbeit mit geflüchteten Kindern und Jugendlichen (S. 12-30). Weinheim: Beltz.

Eubel, C. (2019). Sprachmittlung in der Hilfeplanung. Sozial Extra, 44(2), 92-95. 
Feltes, T., et al. (2017). Zur Kriminalität von Geflüchteten zwischen 2014 und 2016 in NRW. Forschungskonzeption der Analyse der registrierten Kriminalität im Rahmen des Projekts „Flucht als Sicherheitsproblem“. https:// flucht.rub.de/images/arbeitspapiere/arbeitspapier01_flucht_als_sicherheitsproblem.pdf. Zugegriffen: 8. Nov. 2019.

Gruhlich, J. (2019). Schwarze Männlichkeiten. Zur Problematisierung der Problematisierung. In D. Negnal (Hrsg.), Die Problematisierung sozialer Gruppen in Staat und Gesellschaft (S. 43-62). Heidelberg: Springer.

Hörnle, T. (2018). Taten nach $\$ 177$ StGB in der Polizeilichen Kriminalstatistik. Zusammenhänge mit Zuwanderung. Kriminalpolitische Zeitschrift, 3(4), 218-223.

ICMPD (2019). Lebensperspektiven von minderjährigen und jungen erwachsenen Asyl- und subsidiär Schutzberechtigten: Folgerungen für die Integrationspolitik - Ergebnisse der PERSPEKT Studie. Wien: ICMPD.

ISMU (2019). At a crossroad. Unaccompanied and separated children in their transition to audulthood in Italy. https://www.unicef.org/eca/media/8591/file/report-unaccompanied-italy.pdf. Zugegriffen: 8. Sept. 2020

Kury, H. (2018). Migration in Germany: an international comparison on the psychotraumatic stress among refugees. In H. Kury \& S. Redo (Hrsg.), Refugees and migrants in law and policy. Challenges and opportunities for global civic education, (Bd. 17, S. 313-354). Cham: Springer.

LAG Mobile Jugendarbeit (2019): Solidarität statt Ausgrenzung - Mehr Unterstützung und Begleitung für junge Geflüchtete. [https://www.lag-mobil.de/download/solidaritaet-statt-ausgrenzung-mehr-unterstuetzung-undbegleitung-fuer-junge-gefluechtete/]

Lechner, C., \& Huber, A. (2017). Ankommen nach der Flucht. Die Sicht begleiteter und unbegleiteter junger Geflüchteter auf ibre Lebenslagen in Deutschland. München: Deutsches Jugendinstitut.

Pfeiffer, C., Baier, D., \& Kliem, S. (2018). Zur Entwicklung der Gewalt in Deutschland. Schwerpunkte: Jugendliche und Flüchtlinge als Täter und $\mathrm{Op}$ fer. Zürich: Institut für Delinquenz und Kriminalprävention.

Schartau, L. K., et al. (2018). Die Angst vor dem Fremden. Stand der Forschung zu Kriminalitätsfurcht und Unsicherheitswahrnehmungen im Kontext von Migration und Flucht. https://flucht.rub.de/images/arbeitspapiere/Arbeitspapier-3_FluchtalsSicherheitsproblem.pdf. Zugegriffen: 8. Aug. 2020.

Scherr, A., \& Breit, H. (2020). Diskriminierung, Anerkennung und der Sinn für die eigene soziale Position. Wie Diskriminierungserfahrungen Bildungsprozesse und Lebenschancen beeinflussen. Wiesbaden: Springer.

Scherr, A., \& Sachs, L. (2020). „Geflüchtete - Demokrat”innen von Anfang an". Hinweise und Empfehlungen für die Praxis der offenen Kinderund Jugendarbeit. https://www.agif.de/files/cto layout/Material/Aktuelles/ AGJF/Abschlussbericht $\% 20 \mathrm{Ba}-\mathrm{W} \% \mathrm{C} 3 \% \mathrm{BC} \% 20$ und $\% 20$ Sachsen_final. pdf. Zugegriffen: 21. Sept. 2020.

Schweitzer, H. (2019). Management by sherpa. Sozial Extra, 44(2), 88-91. 